\title{
A Preliminary Study of Physical Fitness in 8- to 10-Year-Old Primary School Children From North East England in Comparison With National and International Data
}

\author{
Kathryn L. Weston \\ Teesside University \\ Nicoleta Pasecinic and Laura Basterfield \\ Newcastle University
}

\begin{abstract}
Purpose: Despite recent updates to international normative values for physical fitness in young people, contemporary data sets from England are sparse with no published data available from the North East. We compared physical fitness in children from one primary school in North East England to International and European reference data, and other English regions. Methods: Eighty participants (mean age [SD]: 9.1 [0.6] y) completed a testing battery of 20-m shuttle run test, handgrip strength, standing broad jump, and sit-and-reach. Scores for each component were assessed against International or European age- and sex-specific centiles, then grouped into quintiles. Differences between our sample and European and English data sets were explored using $z$ scores and $t$ tests. Results: For all components, $\geq 58 \%$ of participants were classified as having "moderate" or lower levels. Twenty-meter shuttle run test performance was not substantially different compared with other English data sets. For handgrip and sit-and-reach, our sample scored significantly worse than South East children. Standing broad jump distance in girls, and handgrip in boys and girls, was significantly lower than North West equivalents. Conclusion: Physical fitness levels in primary school children from North East England are suboptimal, highlighting a need for large-scale monitoring studies to build on our preliminary findings.
\end{abstract}

Keywords: fitness testing, muscular fitness, aerobic fitness, young people

Physical fitness can be defined as a set of characteristics related to health and performance, including aerobic fitness, muscular endurance, strength and power, body composition, flexibility, balance, agility, and reaction time (7,22). In children and adolescents, the characteristics directly attributable with health improvements are aerobic fitness, body composition, flexibility, and muscular fitness-a collective term representing muscular strength, local muscular endurance, and muscular power (39)—-(7,20,21,47). Of these, aerobic fitness-often defined as the ability of the working muscle to receive and utilize oxygen for energy production during exercise (4) - is a strong summative marker of physical health (30); with peak oxygen uptake $\left(\mathrm{VO}_{2}\right.$ peak $)$ - the highest rate at which oxygen can be consumed during exercise (4) - widely recognized as the best single measure of aerobic fitness in young people (5). As

(C) 2019 The Authors. Published by Human Kinetics, Inc. This is an Open Access article distributed under the terms of the Creative Commons Attribution License CC BY 4.0, which permits unrestricted noncommercial and commercial use, distribution, and reproduction in any medium, provided the original work is properly cited, the new use includes a link to the license, and any changes are indicated. See https:// creativecommons.org/licenses/by/4.0/. This license does not cover any third-party material that may appear with permission in the article.

Weston is with the School of Health \& Social Care, Teesside University, Middlesbrough, United Kingdom. Pasecinic and Basterfield are with the Human Nutrition Research Centre, Newcastle University, Newcastle upon Tyne, United Kingdom. Basterfield is also with the Institute for Health and Society, Newcastle University, Newcastle upon Tyne, United Kingdom. Basterfield (laura.basterfield@ newcastle.ac.uk) is corresponding author.
$\mathrm{VO}_{2}$ peak is highly correlated with body mass, this is typically controlled for by dividing peak $\mathrm{VO}_{2}(\mathrm{~mL} / \mathrm{min})$ by body mass in $\mathrm{kg}$ and expressing it as a ratio of $\mathrm{mL} / \mathrm{kg} / \mathrm{min}$ (4). Higher levels of aerobic fitness are associated with reduced risk of future metabolic and cardiovascular disease; obesity and mortality $(9,38)$; better mental health (30); and potentially higher levels of academic achievement (19). There is also accumulating evidence linking higher levels of muscular fitness with various physical and psychological health benefits in young people (39), some of which are independent of aerobic fitness.

Despite the well-documented benefits of higher physical fitness in youth, debate remains over whether aerobic fitness in young people has declined over time $(16,33,45)$. This may be partly due to the way different researchers operationalize the term aerobic fitness (4). When aerobic fitness is defined as mass-related $\mathrm{VO}_{2}$ peak, there appears to very little historic change in young people's aerobic fitness (4). Conversely, when the term is operationalized as maximal field-based endurance running performance (eg, 20-m shuttle run test [20mSRT] performance), the global picture is one of decreasing levels for several decades $(4,6,33,41,44,46,50)$. This public health problem is of particular concern in England, with data from some regions indicating that aerobic fitness performance specifically is declining by around $8 \%$ per decade $(36,41)$ - twice the rate observed in other developed nations (45). The issue is further complicated by a lack of contemporary data sets from different English regions. There are only a few data sets available for healthy English children, with large-scale studies concentrated in the East of the country through the East of England Healthy 
Hearts Study $(11,35,50)$ and the Chelmsford Children's Fitness and Activity Survey $(10,37)$, and in the South East by the Camden Active Spaces project (1). The North West is well represented by the repeat cross-sectional study SportsLinx $(6,40,41)$. To date, however, there are no published data on children from the North East of England, who have some of the highest obesity levels in the country (25) and often reside in areas with high levels of deprivation (15). A lack of data in this region is particularly surprising in light of recommendations from the UK's chief medical officer almost a decade ago for comprehensive fitness testing to be introduced in schools (17).

Across the published data sets on English youth, inconsistencies exist with regard to testing procedures (eg, tests administered and time of year of data collection) and the study sample (eg, participants' age and socioeconomic status). Data sets from the East of England, for example, involved participants from rural and urban areas where levels of deprivation were lower than the English national average (10,50), whereas studies in the South East and North West are more representative of participants from urban areas with higher levels of deprivation (Camden and Liverpool) (15). With these caveats in mind, it is unknown whether these data sets are generalizable to young people residing in other geographical areas in England. Recently, youth-specific International and European normative values for physical fitness components have been updated $(42,43)$, which will aid surveillance and monitoring at a global, national, and individual level. To develop a more complete picture of physical fitness levels in English youth, however, all geographical regions of the country must be represented. The aim of our preliminary study therefore was to assess physical fitness levels in 8- to 10-year-old children from one primary school in North East England and compare these to International and European reference data, and other regions in England.

\section{Methods}

\section{Participants and Study Design}

Newcastle University Faculty of Medical Sciences Ethics Committee granted ethics approval for the study. The study is part of a wider pilot study investigating associations between quality of life, physical fitness, and body composition in 8- to 10-year-old primary school children (The Sitting, Outdoor play and Fitness [SOFit] study). The Sitting, Outdoor play and Fitness study assessed children's quality of life, access to outdoor play, electronic games usage and involvement in sports clubs using questionnaires, and physical fitness using the methods outlined below. The current study focuses on the physical fitness variables; the associations between quality of life, outdoor play, and fitness variables will be reported elsewhere. Using a cross-sectional design, all children in the year 4 and year 5 cohorts $(n=82)$ of one school in Gateshead, North East England, were invited to take part. Gateshead is a relatively deprived borough (73/326 local authorities in England where $1=$ most deprived), and the school catchment includes areas in the $10 \%$ to $20 \%$ most deprived areas of the country (15). We used the pupil premium as a proxy for socioeconomic status; the premium is additional funding available to publicly funded schools in England to help minimize the gap between disadvantaged pupils and their peers.

The head teacher provided written informed consent for the school's participation. Parents received full information about the study and the option to opt out their child. Two parents elected not to let their child take part. Participating children $(n=80)$ provided written informed assent prior to formal study enrollment.

\section{Outcome Measures}

The study took place during the winter school term. All testing was conducted indoors in the hard floor school sports hall. Physical fitness was measured using the following components of the Eurofit testing battery (13): 20mSRT performance, handgrip strength, standing broad jump, and sit-and-reach performance.

Anthropometry. Participants' body mass, standing height, and sitting height were measured to the nearest $0.1 \mathrm{~kg}$ and $0.1 \mathrm{~cm}$ using calibrated scales (Shekel H151-7, Shekel Scales LTD; Lower Galilee, Israel) and a portable stadiometer (Leicester Height Measure, SECA UK LTD, Birmingham, England), respectively. Two measurements were taken for each variable, then averaged for analysis. During assessments, participants wore light indoor clothing and were barefoot. Participants' body mass index (BMI) and BMI $z$ score were calculated relative to UK 1990 reference data (12). Leg length was calculated by subtracting sitting height from stature. Somatic maturity was estimated for each participant by predicting years from attainment of peak height velocity via sex-specific multivariable equations that included stature, sitting height, leg length, body mass, chronological age, and their interactions (27). Participants were then classified as either prepeak or postpeak height velocity for analysis.

20-m Shuttle Run Test Performance. Aerobic fitness was indirectly assessed via 20mSRT performance using the British National Coaching Foundation protocol (32). With the aid of a pacer, participants were encouraged to run between cones in time with an audible bleep signal for as long as possible and told they would be asked to stop if they failed to maintain the specified pace for 2 consecutive shuttles. Participants were also allowed to drop out at their own volition at any time if they felt unable to maintain the required pace. Test performance was expressed as the number of shuttles completed.

Handgrip Strength. Handgrip strength was assessed using a hydraulic hand dynamometer (Jamar 5030J1; Hydraulic Hand Dynamometer, Sammons Preston, Chicago, IL). Participants performed the test in a standing position, with the wrist neutral and elbow of the testing arm completely extended and without touching any other part of the body (34). Using their dominant hand, participants were asked to squeeze the dynamometer gradually and continuously for at least 3 seconds. Elbow flexion from $180^{\circ}$ to $90^{\circ}$ was permitted (10). Following a recovery period of at least 3 minutes, the test was repeated. The maximum score (recorded in $\mathrm{kg}$ ) was recorded for analysis.

Standing Broad Jump. Lower body strength was measured via standing broad jump performance. Here, participants were instructed to stand behind a starting line marked out by cones in the sports hall and, with their feet together, jump forward as far as possible. Participants performed 3 practice jumps, followed by 3 measured attempts. For an attempt to be valid, participants had to land with their feet together and remain upright. The distance jumped was measured from the starting line to where the back of the heel nearest to the starting line landed (8) using a tape measure, with the maximum score (recorded in $\mathrm{cm}$ ) retained for analysis.

Sit-and-Reach Performance. Sit-and-reach performance was measured using a steel sit-and-reach box. Participants were required to sit on the sports hall floor with their legs straight and feet against the box. They were then asked to reach out with both hands as far as possible. Three attempts were permitted with the maximum score (recorded in $\mathrm{cm}$ ) retained for analysis. 


\section{Statistical Analysis}

Comparisons with published reference data were completed in 2 ways. First, $z$ scores were created according to sex- and age-specific normative values from the Europe-wide IDEFICS (Identification and prevention of Dietary- and lifestyle-induced health EFfects In Children and infantS) study $(3,14)$. Data were available for handgrip strength and standing broad jump (M. Zaqout, personal communication, 2018). The sit-and-reach protocol for IDEFICS was different to that in our study; therefore, $z$ scores were not created for this variable. Although IDEFICS used the mean of the handgrip scores rather than the maximum, we considered this data suitable to create the $z$ score. Shuttle run $z$ scores were created using the East of England Healthy Hearts Study data, available from UK Data Archive Study Number 7456 (49), for 9- and 10-year-olds. Second, participants' scores for each fitness component were categorized into age- and sex-specific centiles relative to International normative data-20mSRT (43) — and European normative data-handgrip strength, standing broad jump, and sit-and-reach tests (42) - in those aged $\geq 9$ years old. For participants aged 9.0 to $\leq 9.99$ years and aged $\geq 10.0$ years, sex-specific age 9 -year and age 10 -year centile data, respectively, were used. For participants aged $<9$ years, reference standards from the IDEFICS study (14) for boys and girls aged 8 to $<8.5$ years and 8.5 to $<9$ years were used. The exception to this was sit-and-reach data for participants aged $<9$ years as the protocol utilized in the IDEFICS study differed substantially from our own. In the absence of other robust normative values for this subpopulation, sit-and-reach data from our participants aged $<9$ years could not be included in this section of the analysis $(n=17)$.

Following the recommendations of Tomkinson et al $(42,43)$, a quintile framework was adopted for reporting purposes. Here, participants in centiles below 20 were categorized as having "very low" levels of a fitness outcome, "low" levels for scores between the 20th and 40th centiles, "moderate" levels for scores between the 40th and 60th centiles, "high" for scores between the 60th and 80th centiles, and "very high" levels for scores above the 80th centile. For each fitness measure, we report the frequency and percentage of participants in each quintile. For the exact scores corresponding to the centile for each fitness outcome, please see Tomkinson et al (43) (20mSRT for participants aged
9.0 to $>10 \mathrm{y}$ ); Tomkinson et al (42) (handgrip strength, standing broad jump, and sit-and-reach for participants aged 9.0 to $>10 \mathrm{y}$ ); and De Miguel-Etayo et al (14) (20mSRT, handgrip strength, and standing broad jump for participants aged $<9.0 \mathrm{y}$ ).

To determine whether physical fitness was predicted by BMI, associations between participants' BMI/BMI $z$ score, sex, age, and physical fitness variables were tested with linear regression using SPSS Statistics software (v.21; IBM Corp, Armonk, NY). Where $z$ scores were available, these were used to eliminate the need to adjust for age and sex. Beta $(B)$ coefficients with $95 \%$ confidence intervals (CIs) are reported. All participants were classed as prepeak height velocity; therefore, this variable was not used in analyses. To assess differences between our data and other published studies conducted in England, summary data of mean and SD (where available) were used for 2-sample $t$ tests in STATA 15 (v.15; Stata Corp, College Station, TX) using the "immediate" command. Significance was set at $P<.05$.

Of the 80 participants who volunteered to take part in the study, one was unable to complete any assessments other than handgrip strength on medical grounds. For the $20 \mathrm{mSRT}, 3$ children were absent for testing and one participant abstained due to a preexisting injury. Two children were absent for height and weight measurements, with one other child unavailable for weight measurement only. Six participants were absent for standing broad jump, and 9 were absent for sit-and-reach. One participant was absent for the handgrip strength test.

\section{Results}

\section{Descriptive and Summary Data}

Participants' descriptive data (mean [SD]) and the number of participants assessed for each physical fitness outcome are in Table 1. The mean age of the participants was 9.1 years, with a BMI $z$ score of 0.51 . On average, boys completed 37 shuttles compared with 27 by girls. Boys jumped on average $128.6 \mathrm{~cm}$ compared with $114.5 \mathrm{~cm}$ for girls, and reached $13.6 \mathrm{~cm}$ compared with girls' $16.3 \mathrm{~cm}$. Mean handgrip strength of the sample was $13.3 \mathrm{~kg}$ (Table 1).

Table 1 Participants' Descriptive Characteristics

\begin{tabular}{|c|c|c|c|c|c|c|}
\hline \multirow[b]{2}{*}{ Variable } & \multicolumn{6}{|c|}{ Participants, mean (SD) or mean $(95 \% \mathrm{CI})$} \\
\hline & Boys & $\mathbf{n}$ & Girls & $\mathbf{n}$ & All & $\mathbf{N}$ \\
\hline Age, y & $9.3(0.6)$ & 35 & $9.0(0.5)$ & 45 & $9.1(0.6)$ & 80 \\
\hline Age $z$ score $^{\mathrm{a}}$ & $0.2(-0.1$ to 0.5$)$ & & $-0.2(-0.4$ to 0.1$)$ & & - & \\
\hline BMI, kg/m² & $17.3(2.6)$ & 33 & $18.4(3.4)$ & 44 & $17.9(3.1)$ & 77 \\
\hline BMI $z$ score & $0.39(1.05)$ & 33 & $0.61(1.20)$ & 44 & $0.51(1.14)$ & 77 \\
\hline 20mSRT, $\mathrm{n}$ & $37(11)$ & 32 & $27(10)$ & 43 & $31(11)$ & 75 \\
\hline $20 \mathrm{mSRT} z$ score $^{\mathrm{a}}$ & $-0.1(-0.4$ to 0.1$)$ & & $-0.1(-0.4$ to 0.2$)$ & & - & \\
\hline Handgrip, kg & $13.9(3.2)$ & 34 & $12.9(2.8)$ & 45 & $13.3(3.0)$ & 79 \\
\hline Handgrip $z$ score $^{\mathrm{a}}$ & $-0.2(-0.6$ to 0.2$)$ & & $-0.0(-0.3$ to 0.3$)$ & & - & \\
\hline Broad jump, $\mathrm{cm}$ & $128.6(18.3)$ & 30 & $114.5(17.8)$ & 43 & $120.3(19.2)$ & 73 \\
\hline Broad jump $z$ score $^{\mathrm{a}}$ & $0.2(-0.1$ to 0.5$)$ & & $-0.2(-0.4$ to 0.1$)$ & & - & \\
\hline Sit-and-reach, cm & $13.6(5.6)$ & 27 & $16.3(6.1)$ & 43 & $15.3(6.0)$ & 70 \\
\hline
\end{tabular}

Abbreviations: BMI, body mass index; CI, confidence interval; IDEFICS, Identification and prevention of Dietary- and lifestyle-induced health EFfects in Children and infantS; 20mSRT, 20-m shuttle run test.

${ }^{a}$ Reference group for 20mSRT performance: East of England Healthy Hearts Study (49), data available for ages 9 and 10 only; $\mathrm{n}=378$ boys and 351 girls. Reference group for age, handgrip strength, and standing broad jump distance: IDEFICS (14); $\mathrm{n}=2602$ boys and 2768 girls. No reference group for sit-and-reach. 


\section{Outcome Data}

Based on UK reference BMI data (12), 70\% of participants were healthy weight, $12 \%$ were overweight, and $18 \%$ were obese. This is lower than the North East values for the slightly older children in the National Child Measurement Programme (English 10- to 11-y-olds) of $14.6 \%$ overweight ( $95 \%$ CI, 14.2 to 15.0$)$ and $22.4 \%$ obese $(95 \%$ CI, 21.9 to 22.9), and for Gateshead of $15.0 \%$ overweight $(95 \% \mathrm{CI}$, 13.4 to 16.7 ) and $22.8 \%$ obese (95\% CI, 20.9 to 24.7) (28). In 2015$2016,27.5 \%$ of pupils at our participants' school were eligible for the pupil premium; higher than the English average of $25.8 \%(95 \% \mathrm{CI}$, 25.5 to 26.0), but lower than the North East average of $32.4 \%$ (95\% CI, 29.5 to 36.9) and Gateshead $31.0 \%$ (18).

Participants' physical fitness scores expressed in quintiles corresponding to "very low," "low," "moderate," "high," and "very high" levels $(42,43)$ are shown in Figure 1. For 20mSRT performance, $12 \%(\mathrm{n}=9 ; 4$ boys), $21 \%(\mathrm{n}=16 ; 3$ boys), and $25 \%$ ( $n=19$; 9 boys) of participants' scores fell in the "very low," "low," and "moderate" quintiles, respectively. Of the remainder, 20\% $(\mathrm{n}=15 ; 10$ boys $)$ scored in the "high levels" quintile and $21 \%(\mathrm{n}=16$; 6 boys) in the "very high" levels (Figure 1). Linear regression analysis revealed no statistically significant association between age and 20mSRT performance. There was an influence of sex on number of shuttles run, with boys running more than girls $(P<.001$, Table 2). BMI and BMI $z$ score were both strong predictors of 20mSRT $z$ score, with children of higher body mass running fewer shuttles (Table 2). There was no effect of sex on BMI.

For handgrip strength, 29\% $(n=23 ; 13$ boys $), 27 \%(n=21 ; 7$ boys), and $14 \%(\mathrm{n}=11 ; 7$ boys) of participants scored in the "very low," "low," and "moderate" quintiles, respectively; $25 \%$ ( $n=20$; 7 boys) and $5 \%(\mathrm{n}=4 ; 1$ boy) of participants' scores fell in the "high" or "very high" levels quintiles. There were no sex differences in handgrip strength and no association of handgrip $z$ scores with BMI (Table 2). Handgrip strength was predicted by age
$(P=.001$, Table 2$)$. With regard to standing broad jump performance, $33 \%$ ( $\mathrm{n}=24 ; 6$ boys), $23 \%$ ( $\mathrm{n}=17 ; 9$ boys), and $23 \%$ ( $n=17$; 9 boys) of participants' scores fell in the "very low," "low," and "moderate" quintiles, respectively; $19 \%(\mathrm{n}=14 ; 6$ boys) and $1 \%$ ( $\mathrm{n}=1 ; 0$ boys) of participants, respectively, recorded scores which placed them in the "high" and "very high" quintiles. There were significant effects of sex on jump distance; boys jumped further than girls $(P=.002$, Table 2). Jump distance $z$ score was significantly associated with BMI and BMI $z$ score (Table 2). There was no significant effect of age on jump distance.

For sit-and-reach, $50 \%$ ( $\mathrm{n}=27 ; 13$ boys) of participants analyzed scored in the "very low" quintile; $9 \%$ ( $n=5 ; 3$ boys), $26 \%$ ( $\mathrm{n}=14 ; 5$ boys), $13 \%$ ( $\mathrm{n}=7 ; 3$ boys), and $2 \%(\mathrm{n}=1 ; 1$ boy) of participants" scores fell in the "low," "moderate," "high," and "very high" quintiles, respectively. There was a significant effect of sex on sit-and-reach performance; girls recorded higher scores than boys $(P=.02$, Table 2$)$. There was no association with age, BMI, or BMI $z$ score.

\section{Comparisons With Other English Regions}

When our data were compared to European (IDEFICS) and the East of England Healthy Hearts Study reference data $(14,49)$ using $z$ scores, there were no meaningful differences in physical fitness parameters between the children in our study and the age- and sexmatched reference populations ( $z$ scores shown in Table 1$)$. Table 3 details study level and participant characteristics from our investigation and other contemporary English data sets where data were available $(1,6,10,11,35,37,40)$. As publicly available raw data were not available for English data other than from East of England Healthy Hearts Study, further statistical comparisons of physical fitness parameters via $z$ scores were not possible. Narratively, 20mSRT performance in our 8- to 10-year-old sample from the North East was slightly higher for boys (median 36 vs 33 shuttles)

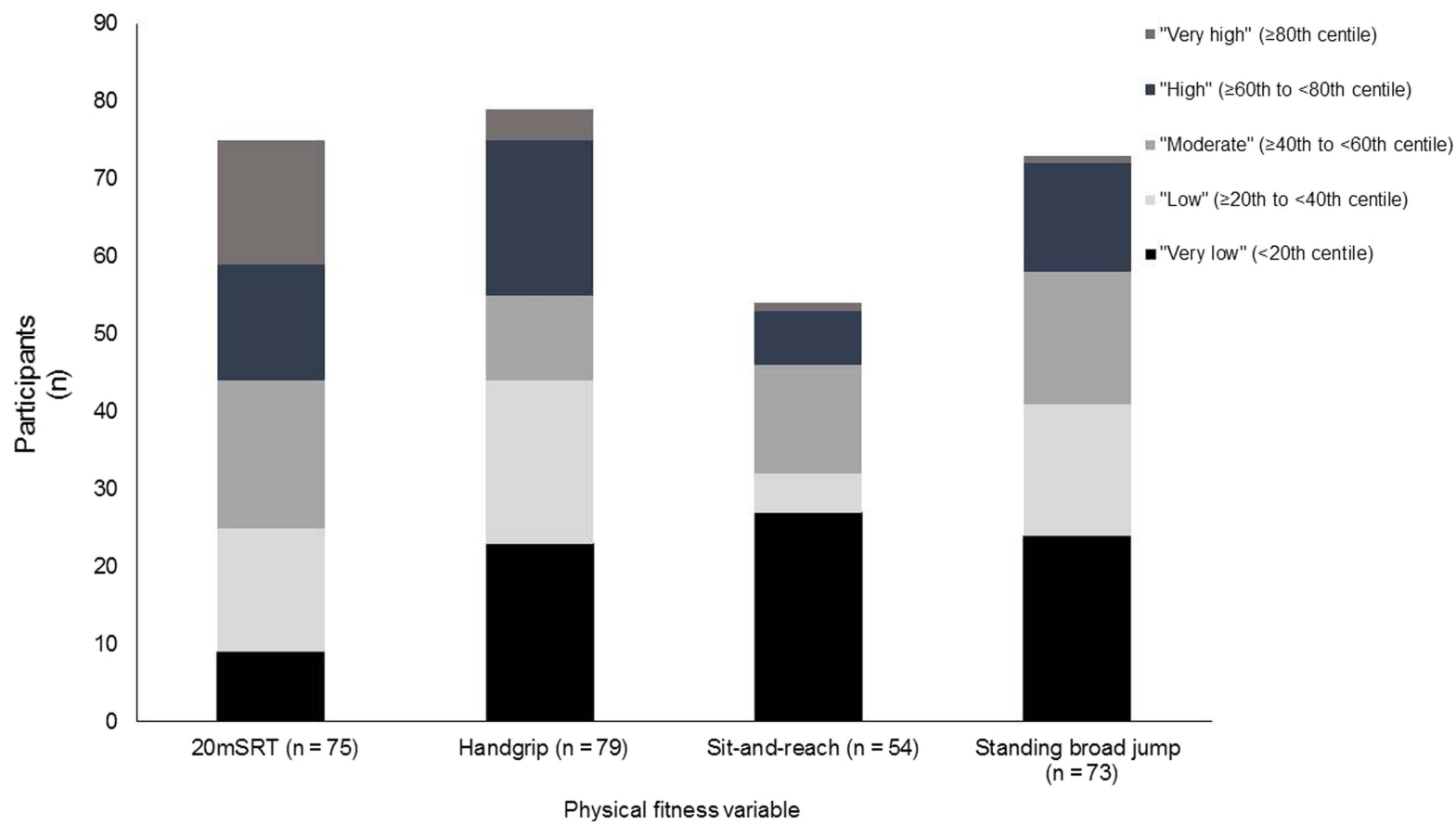

Figure 1 - Quintile classification framework for physical fitness components, based on International (43) and European normative values (42). For each physical fitness outcome measure, the number of participants included in the analysis is shown in brackets. 20mSRT indicates 20 -m shuttle run test performance. 
Table 2 Univariate Associations Between Physical Fitness, Age, Sex, and Body Composition Variables

\begin{tabular}{|c|c|c|c|c|}
\hline Dependent/independent variables & $B$ coefficient & $95 \%$ confidence interval & Adjusted $R^{2}$ & $P$ \\
\hline \multicolumn{5}{|l|}{ Dependent: 20mSRT performance } \\
\hline Age, y & 3.093 & -1.477 to 7.663 & .011 & .18 \\
\hline Sex & -9.882 & -14.649 to -5.115 & .178 & $<.001$ \\
\hline \multicolumn{5}{|l|}{ Dependent: handgrip strength } \\
\hline Age, y & 1.870 & 0.745 to 2.995 & .113 & .001 \\
\hline Sex & -1.016 & -2.381 to 0.349 & .015 & .14 \\
\hline \multicolumn{5}{|l|}{ Dependent: broad jump } \\
\hline Age, y & 3.464 & -4.530 to 11.459 & -.004 & .39 \\
\hline Sex & -14.032 & -22.586 to -5.478 & .119 & .002 \\
\hline \multicolumn{5}{|l|}{ Dependent: sit-and-reach } \\
\hline Age, y & -2.529 & -5.149 to 0.092 & .037 & .06 \\
\hline Sex & 3.659 & 0.673 to 6.645 & .065 & .02 \\
\hline \multicolumn{5}{|l|}{ Dependent: $20 \mathrm{mSRT} z$ score } \\
\hline BMI & -0.094 & -0.160 to -0.028 & .149 & .01 \\
\hline BMI $z$ score & -0.265 & -0.442 to -0.089 & .167 & .004 \\
\hline \multicolumn{5}{|l|}{ Dependent: handgrip $z$ score } \\
\hline BMI & 0.036 & -0.041 to 0.112 & -.002 & .35 \\
\hline BMI $z$ score & 0.140 & -0.069 to 0.349 & .010 & .19 \\
\hline \multicolumn{5}{|l|}{ Dependent: broad jump $z$ score } \\
\hline BMI & -0.094 & -0.156 to -0.032 & .104 & .004 \\
\hline BMI $z$ score & -0.220 & -0.391 to -0.049 & .074 & .01 \\
\hline \multicolumn{5}{|l|}{ Dependent: age $z$ score } \\
\hline BMI & -0.018 & -0.084 to 0.048 & -.009 & .58 \\
\hline BMI $z$ score & -0.073 & -0.254 to 0.108 & -.005 & .42 \\
\hline
\end{tabular}

Abbreviations: BMI, body mass index; 20mSRT, 20-m shuttle run test.

and lower for girls (median 24 vs 27 shuttles) than older participants (mean age: $10.4 \mathrm{y}$ ) in the East of England from the Chelmsford Children's Fitness and Activity Survey $(10,35)$. Our North East children ran fewer shuttles than counterparts in the North West, but there was no statistical difference from age-matched children in the East of England Healthy Hearts study (Table 3). For handgrip strength, our North East sample (boys and girls) scored significantly worse $(P<.001)$ than their North West counterparts from the 20042005 data set and children in the South East $(P=.002)$. Differences between our sample of North East boys and girls and both data sets from the East were not statistically significant (Table 3). Standing broad jump performance did not differ significantly in our sample of North East boys compared with North West boys in 2004-2005 or South East children collectively, but performance in our sample of North East girls was significantly worse $(P<.001)$ than North West girls from 2004 to 2005 (Table 3). For sit-and-reach performance, our sample of North East boys and girls scored similar to their North West counterparts from 2004 to 2005, but lower than the South East participants combined $(P<.001)$ (Table 3$)$.

\section{Discussion}

Published data sets on physical fitness levels in English school children are sparse and in some regions of the country, completely absent. The aim of our study therefore was to assess physical fitness components in 8- to 10-year-old children from one primary school in Gateshead, North East England, against International and European age- and sex-specific normative values $(42,43)$, and compare our data to other contemporary youth studies in England. In doing so, we have provided the first published data set from the North East region on 8- to 10-year-old primary school children's physical fitness levels, which we hope will aid more comprehensive insights into physical fitness levels in youths from different geographical locations in England.

The use of $z$ scores indicated that, when compared with sexand age-matched data from European children in the IDEFICS study (14), and children in the East of England Healthy Hearts Study (49), there were no meaningful differences in most physical fitness parameters. We were unable to compare sit-and-reach, as differences in testing protocols negated comparisons between our sample and the IDEFICS data, and data for this variable were not available from the East of England Healthy Hearts Study (49). Against sex-specific age 9- and 10-year International normative $20 \mathrm{mSRT}$ values for number of shuttles completed (43), $58 \%$ of our participants $(n=44)$ had "moderate" $(n=19)$, "low" $(n=16)$, or "very low" $(\mathrm{n}=9)$ levels of $20 \mathrm{mSRT}$ performance. Boys performed more shuttles than girls, which is in line with observations from large pooled international data sets (43). BMI and BMI $z$ score were both strong predictors of 20mSRT performance, with children of higher body mass running fewer shuttles. This is line with observations that aerobic fitness performance is affected by increased fat mass (4). Currently, however, it is unknown whether children of higher body mass completing fewer shuttles is due to a lower aerobic fitness per se, or because they are carrying a higher body mass and therefore do more work in every shuttle. To elucidate this further would require a direct assessment of $\mathrm{VO}_{2}$ peak 


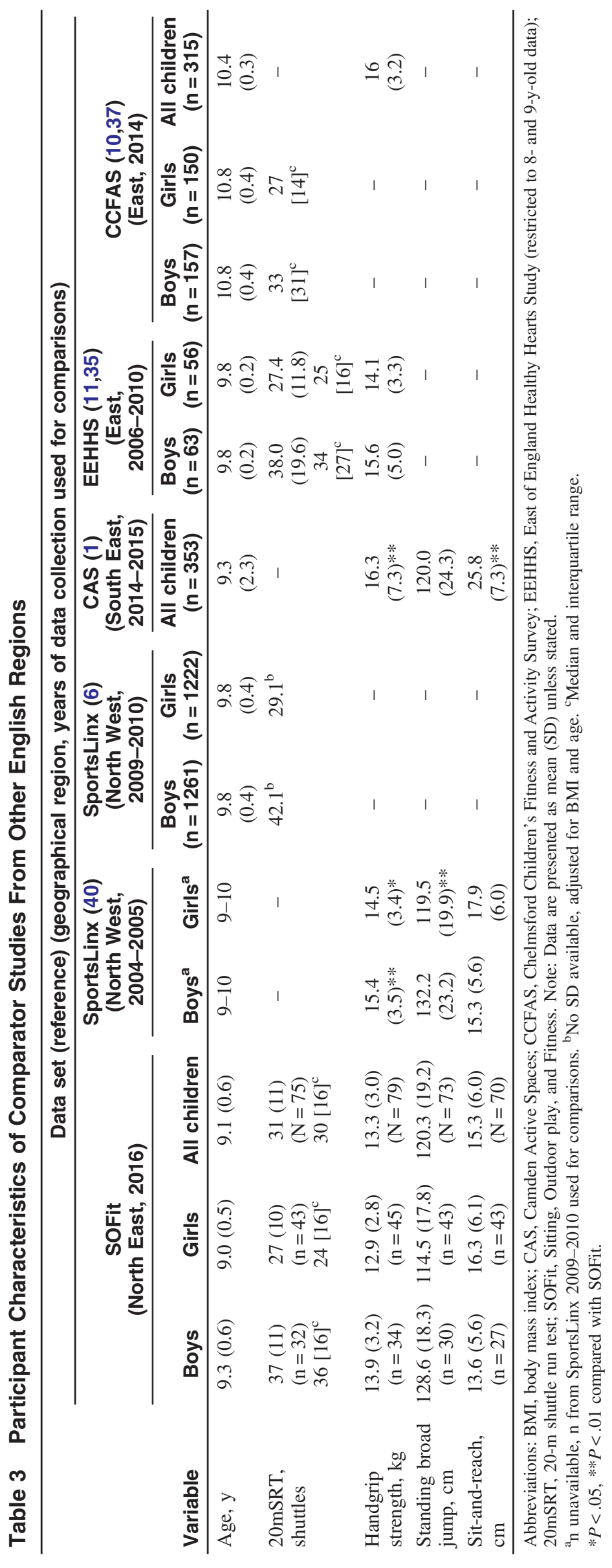


scaled relative to an individual's body mass, or even fat-free mass (26), to be compared with $20 \mathrm{mSRT}$ performance, for example, which was beyond the scope of the current study.

Participants' 20mSRT scores were distributed relatively evenly across the quintile scoring framework (43). Despite a lack of publicly available raw data for comparator English data sets other than the East of England Healthy Heart Study limiting more robust statistical comparisons (eg, creation of a $z$ score based on all English data rather than only one region), it appears that 20mSRT performance in our sample of 8- to 10-year-old children from the North East did not differ substantially from other English regions. This finding is encouraging, given our participants were from a relatively deprived area compared with the English average, and in light of recent suggestions that higher levels of aerobic fitness can reduce or negate the association between deprivation and obesity (29). It is, however, important to highlight that these observations should only currently be viewed as preliminary in nature, due to the small sample size of our study.

Considering longitudinal data revealing negative associations between decreasing muscular strength from childhood to adolescence and changes in adiposity $(24,48)$, it is potentially concerning that $70 \%$ and $79 \%$ of North East participants' handgrip strength and standing broad jump performances, respectively, were classified as "moderate" or below (42). Furthermore, 29\% of participants' handgrip scores fell in the "very low" level quintile, with both boys and girls from our sample recording significantly worse scores than their North West counterparts from 2004 to 2005 (40) and children in South East (1). It should be noted that although the grip size of our dynamometer was on the smallest setting, hand span measurements and subsequent grip size adjustments were not conducted due to time restrictions, which may have impacted on the scores recorded (34). In contrast with observations from large pooled European data sets (42), there were no significant differences in scores between boys and girls for handgrip strength. For standing broad jump and sit-andreach performance, boys jumped significantly further than girls, and girls reached significantly further than boys, respectively, which is in line with European data set trends (42). Against European normative values for standing broad jump, 33\% of our sample of North East children scored within the "very low" level quintile, and when compared with their North West counterparts from the 20042005 data set, standing broad jump distance in our sample of girls was significantly worse. While this is no doubt worrying, standing broad jump can be a very technical test (8), with performance dependent on factors such as mechanics, coordinative abilities, and anthropometrics $(2,31)$. This may partly explain why participants with lower BMI and BMI $z$ scores jumped further than those with higher BMI data.

Collectively, the contrasting findings for different fitness components within our sample reiterate the need for multifaceted testing batteries in youth (39), especially when assessing the effectiveness of interventions aimed at improving multiple fitness aspects. Although our study has provided the first data set on physical fitness levels in 8- to 10-year-old primary school children from one school in North East England, it is not without limitations. Given the relatively small sample size and tight age range of participants drawn from only one school, our findings cannot be generalized to children across all of North East England at this time. Despite being in a region of relative deprivation, the school has a lower uptake of free school meals than others in the region, but still more than the English average. BMI data and overweight and obesity levels in our study are slightly lower than those observed in older children across the North East Region (25).
Also, as our study focused on 8- to 10-year-old primary school children, physical fitness levels in North East adolescents remain relatively unknown. Although $20 \mathrm{mSRT}$ performance (51) and several muscular fitness variables (vertical jump height and handgrip strength) (23) have been measured in recent North East-based exercise investigations involving adolescents, interpretation of these data are complicated by the interventional nature of some of these studies. Finally, as comparisons with other English data sets were often limited by missing data and the use of different age ranges and reporting techniques, interpretation and extrapolation of these findings should be performed with caution. However, the creation of $z$ scores using European and English normative data where available did indicate that there were no meaningful differences between the children in this study and their age- and sex-matched counterparts.

\section{Conclusion}

In the first published data set on physical fitness variables in 8- to 10 -year-old children from one school in Gateshead, North East England, our preliminary findings indicate that physical fitness levels may be suboptimal, which strongly highlights the need for the implementation of large-scale monitoring studies in the region. Future studies should aim to recruit larger numbers of participants from primary and secondary schools across the North East region of England. Where possible, data should be collected at various time points throughout the school year and repeated longitudinally to enable a rich data set on youth physical fitness trends in the region to be created. Although the use of fitness testing in schools remains a divisive topic (9), our data support the viewpoint that physical fitness testing within the school setting has utility for both sport and health promotion in young people $(42,43)$ from a national and global perspective.

\section{Acknowledgments}

We are extremely grateful to the children, teachers, and parents for their enthusiasm and cooperation. L.B. and K.L.W. are members of Fuse, the Centre for Translational Research in Public Health (www.fuse.ac.uk). Fuse is a UK Clinical Research Collaboration (UKCRC) Public Health Research Centre of Excellence. Funding for Fuse from the British Heart Foundation, Cancer Research UK, Economic and Social Research Council, Medical Research Council, and the National Institute for Health Research, under the auspices of the UKCRC, is gratefully acknowledged. The views expressed in this paper do not necessarily represent those of the funders or UKCRC. The funders had no role in study design, data collection and analysis, decision to publish, or preparation of the manuscript.

\section{References}

1. Aggio D, Smith L, Hamer M. Effects of reallocating time in different activity intensities on health and fitness: a cross sectional study. Int $J$ Behav Nutr Phys Act. 2015;12(1):83. doi:10.1186/s12966-015-0249-6

2. Aguado X, Izquierdo M, Montesinos JL. Kinematic and kinetic factors related to the standing long jump performance. J Hum Mov Stud. 1997;32:157-69.

3. Ahrens W, Bammann K, Siani A, et al. The IDEFICS cohort: design, characteristics and participation in the baseline survey. Int J Obes. 2011;35(Suppl 1):S3-15. doi:10.1038/ijo.2011.30

4. Armstrong N, Tomkinson G, Ekelund U. Aerobic fitness and its relationship to sport, exercise training and habitual physical activity 
during youth. Br J Sports Med. 2011;45(11):849-58. PubMed ID: 21836169 doi:10.1136/bjsports-2011-090200

5. Armstrong N, Welsman JR, Kirby BJ. Peak oxygen uptake and maturation in 12-yr olds. Med Sci Sports Exerc. 1998;30:165-9. PubMed ID: 9475659 doi:10.1097/00005768-199801000-00023

6. Boddy LM, Thomas NE, Fairclough SJ, et al. ROC generated thresholds for field-assessed aerobic fitness related to body size and cardiometabolic risk in schoolchildren. PLOS ONE. 2012;7(9): e45755. PubMed ID: 23029224 doi:10.1371/journal.pone.0045755

7. Caspersen CJ, Powell KE, Christenson GM. Physical activity, exercise, and physical fitness: definitions and distinctions for healthrelated research. Public Health Rep. 1985;100(2):126-31. PubMed ID: 3920711

8. Castro-Piñero J, Ortega FB, Artero EG, et al. Assessing muscular strength in youth: usefulness of standing long jump as a general index of muscular fitness. J Strength Cond Res. 2010;24(7):1810-7. doi:10. 1519/JSC.0b013e3181ddb03d

9. Cohen DD, Voss C, Sandercock GRH. Fitness testing for children: let's mount the zebra! J Phys Act Health. 2015;12(5):597-603. PubMed ID: 24905807 doi:10.1123/jpah.2013-0345

10. Cohen DD, Voss C, Taylor MJD, Delextrat A, Ogunleye AA, Sandercock GRH. Ten-year secular changes in muscular fitness in English children. Acta Paediatr. 2011;100(10):E175-7. PubMed ID: 21480987 doi:10.1111/j.1651-2227.2011.02318.x

11. Cohen DD, Voss C, Taylor MJD, Stasinopoulos DM, Delextrat A, Sandercock GRH. Handgrip strength in English schoolchildren. Acta Paediatr. 2010;99(7):1065-72. PubMed ID: 20178516 doi:10.1111/ j.1651-2227.2010.01723.x

12. Cole TJ, Freeman J, Preece M. Body mass index reference curves for the UK, 1990. Arch Dis Child. 1995;73(1):25-9. PubMed ID: 7639544 doi:10.1136/adc.73.1.25

13. Committee for the Development of Sport Council of Europe. Eurofit: Handbook for the Eurofit Tests of Physical Fitness; Rome, Italy: Council of Europe; 1988.

14. De Miguel-Etayo P, Gracia-Marco L, Ortega FB, et al. Physical fitness reference standards in European children: the IDEFICS study. Int J Obes. 2014;38 Suppl 2:S57-66. PubMed ID: 25376221 doi: 10.1038/ijo.2014.136

15. Department for Communities and Local Government. The English Indices of Deprivation 2015. Department for Communities and Local Government; 2015. https://www.gov.uk/government/ statistics/english-indices-of-deprivation-2015. Accessed September $18,2018$.

16. DiNubile NA. Youth fitness-problems and solutions. Prev Med. 1993;22(4):589-94. PubMed ID: 8415512 doi:10.1006/pmed.1993. 1053

17. Donaldson L. Annual Report of the Chief Medical Officer. London, UK: Department of Health; 2009.

18. Education Funding Agency. Pupil premium: funding allocations 2015 to 2016. 2016. https://www.gov.uk/government/publications/ pupil-premium-2015-to-2016-allocations. Accessed September 18, 2018.

19. Fedewa AL, Ahn S. The effects of physical activity and physical fitness on children's achievement and cognitive outcomes. Res $Q$ Exerc Sport. 2011;82(3):521-35. PubMed ID: 21957711 doi:10. 1080/02701367.2011.10599785

20. Freedson PS, Cureton KJ, Heath GW. Status of field-based fitness testing in children and youth. Prev Med. 2000;31(2):S77-85. doi:10. 1006/pmed.2000.0650

21. Gallahue DL, Ozman JC. Understanding Motor Development: Infants, Children, Adolescents, Adults. 6th ed. Boston, MA: McGraw-Hill; 2006.
22. Howley ET. Type of activity: resistance, aerobic and leisure versus occupational physical activity. Med Sci Sports Exerc. 2001;33(6): S364-9. doi:10.1097/00005768-200106001-00005

23. Ingle L, Stephenson A, Sandercock GR. Physical activity profiles and selected muscular fitness variables in English schoolchildren: a north-south divide? Euro J Sport Sci. 2016;16(8):1187-96. doi:10. 1080/17461391.2016.1183714

24. Janz KF, Dawson JD, Mahoney LT. Increases in physical fitness during childhood improve cardiovascular health during adolescence: the Muscatine study. Int J Sports Med. 2002;23:15-21. PubMed ID: 12012257 doi:10.1055/s-2002-28456

25. Lifestyles Statistics Team Health and Social Care Information Centre. National Child Measurement Programme: England, 2014/ 15 school year. Health and Social Care Information Centre. 2015. https://digital.nhs.uk/data-and-information/publications/statistical/ national-child-measurement-programme/2014-15-school-year.

26. Lolli L, Batterham AM, Weston KL, Atkinson G. Size exponents for scaling maximal oxygen uptake in over 6500 humans: a systematic review and meta-analysis. Sports Med. 2017;47(7):1405-19. PubMed ID: 28058696 doi:10.1007/s40279-016-0655-1

27. Mirwald RL, Baxter-Jones AD, Bailey DA, Beunen GP. An assessment of maturity from anthropometric measurements. Med Sci Sports Exerc. 2002;34(4):689-94. PubMed ID: 11932580

28. National Statistics. National Child Measurement Programme England, 2015/16 school year. 2016. https://www.gov.uk/government/ statistics/national-child-measurement-programme-england-2015-to2016-school-year. Accessed August 21, 2018.

29. Nevill AM, Duncan MJ, Lahart I, Sandercock G. Modelling the association between weight status and social deprivation in English school children: can physical activity and fitness affect the relationship? Ann Hum Biol. 2016;43(6):497-504. PubMed ID: 26515632 doi:10.3109/03014460.2015.1115126

30. Ortega FB, Ruiz JR, Castillo MJ, Sjöström M. Physical fitness in childhood and adolescence: a powerful marker of health. Int J Obes. 2008;32(1):1-11. doi:10.1038/sj.ijo.0803774

31. Pandy MG. Optimal muscular coordination strategies for jumping. J Biomech. 1991;24:1-10. PubMed ID: 2026629 doi:10.1016/00219290(91)90321-D

32. Ramsbottom R, Brewer J, Williams C. A progressive shuttle run test to estimate maximal oxygen uptake. Br J Sports Med. 1988; 22(4):141-4. PubMed ID: 3228681 doi:10.1136/bjsm.22.4.141

33. Rowland T. Declining cardiorespiratory fitness in youth: fact or supposition? Pediatr Exerc Sci. 2002;14:1-8. doi:10.1123/pes.14.1.1

34. Ruiz JR, España-Romero V, Ortega FB, Sjöström M, Castillo MJ, Gutierrez A. Hand span influences optimal grip span in male and female teenagers. J Hand Surg Am. 2006;31(8):1367-72. PubMed ID: 17027801 doi:10.1016/j.jhsa.2006.06.014

35. Sandercock G, Voss C, Cohen DD, Taylor M, Stasinopoulos DM. Centile curves and normative values for the twenty metre shuttle-run test in English schoolchildren. J Sports Sci. 2012;30(7):679-87. PubMed ID: 22339646 doi:10.1080/02640414.2012.660185

36. Sandercock G, Voss C, McConnell D, Rayner P. Ten year secular declines in the cardiorespiratory fitness of affluent English children are largely independent of changes in body mass index. Arch Dis Child. 2010;95(1):46-7. PubMed ID: 20040682 doi:10.1136/adc. 2009.162107

37. Sandercock GR, Ogunleye A, Voss C. Six-year changes in body mass index and cardiorespiratory fitness of English schoolchildren from an affluent area. Int J Obes. 2015;39(10):1504-7. doi:10.1038/ijo. 2015.105

38. Schmidt MD, Magnussen CG, Rees E, Dwyer T, Venn AJ. Childhood fitness reduces the long-term cardiometabolic risks associated with 
childhood obesity. Int J Obes. 2016;40(7):1134-40. doi:10.1038/ijo. 2016.61

39. Smith JJ, Eather N, Morgan PJ, Plotnikoff RC, Faigenbaum AD, Lubans DR. The health benefits of muscular fitness for children and adolescents: a systematic review and meta-analysis. Sports Med. 2014;44(9):1209-23. PubMed ID: 24788950 doi:10.1007/s40279014-0196-4

40. SportsLinx. Childhood Fitness in Liverpool 1998-2006. Liverpool: England, UK; 2009.

41. Stratton G, Canoy D, Boddy LM, Taylor SR, Hackett AF, Buchan IE. Cardiorespiratory fitness and body mass index of 9-11-year-old English children: a serial cross-sectional study from 1998 to 2004. Int J Obes. 2007;31(7):1172-8. doi:10.1038/sj.ijo.0803562

42. Tomkinson GR, Carver KD, Atkinson F, et al. European normative values for physical fitness in children and adolescents aged 9-17 years: results from 2779165 Eurofit performances representing 30 countries. Br J Sports Med. 2018;52(22):1445-63. PubMed ID: 29191931 doi:10.1136/bjsports-2017-098253

43. Tomkinson GR, Lang JJ, Tremblay MS, et al. International normative $20 \mathrm{~m}$ shuttle run values from 1142026 children and youth representing 50 countries. Br J Sports Med. 2017;51(21):1545-54. PubMed ID: 27208067 doi:10.1136/bjsports-2016-095987

44. Tomkinson GR, Leger LA, Olds TS, Cazorla G. Secular trends in the performance of children and adolescents (1980-2000) — an analysis of 55 studies of the $20 \mathrm{~m}$ shuttle run test in 11 countries. Sports Med. 2003;33(4):285-300. PubMed ID: 12688827 doi:10.2165/00007256200333040-00003
45. Tomkinson GR, Olds T, editors. Pediatric Fitness: Secular Trends and Geographic Variability. Basel, Switzerland: Karger Medical and Scientific Publishers; 2007.

46. Tremblay MS, Shields M, Laviolette M, Craig CL, Janssen I, Connor Gorber S. Fitness of Canadian children and youth: results from the 2007-2009 Canadian Health Measures Survey. Health Rep. 2010; 21(1):7-20. PubMed ID: 20426223

47. Trost S. Discussion Paper for the Development of Recommendations for Children's and Youth's Participation in Health Promoting Physical Activity. Canberra, Australia: Australian Department of Health and Ageing; 2005.

48. Twisk JW, Kemper HC, Van M echelen W. Tracking of activity and fitness and the relationship with cardiovascular disease risk factors. Med Sci Sports Exerc. 2000;32:1455-61. PubMed ID: 10949012 doi:10.1097/00005768-200008000-00014

49. Sandercock G. East of England Healthy Hearts Study: CrossSectional Data, 2006-2011. UK Data Service, Study Number 7456; 2014. Accessed July 7, 2018.

50. Voss C, Sandercock GRH. Aerobic fitness and mode of travel to school in English schoolchildren. Med Sci Sports Exerc. 2010;42(2):281-7. PubMedＩD: 20083960 doi:10.1249/MSS. 0b013e3181b11bdc

51. Weston KL, Azevedo LB, Bock S, Weston M, George KP, Batterham AM. Effect of novel, school-based high-intensity interval training (HIT) on cardiometabolic health in adolescents: project FFAB (Fun Fast Activity Blasts) — an exploratory controlled before-and-after trial. PLoS ONE. 2016;11(8):e0159116. doi:10.1371/journal.pone.0159116 